\title{
A Novel Architecture for Real-time Operation of Multi-Agent Based Coordination of Demand and Supply
}

\author{
M.P.F. Hommelberg, B.J. van der Velde, C.J. Warmer, I.G. Kamphuis, J.K. Kok
}

\begin{abstract}
The PowerMatcher concept [1], [2], [3] developed at ECN has proven its value in the coordination of demand and supply of electricity in different settings with respect to distributed generation and accommodation of renewable energy resources. The concept has been applied in several field tests and simulations at various levels in the power system. The agent based technology on which the PowerMatcher is built has a number of advantages above other approaches such as the flexibility of the concept to accommodate a large variety of business scenarios, the autonomy of the agents, the standardization of communication through bids and allocation, the hiding of process information, etc.

Yet the field tests also have identified a number of enhancements that may lead to improved behavior of the PowerMatcher in real life circumstances. Also discussions within the power system agent community as laid down in two White Papers from the IEEE Multi-Agent Systems Working Group [4] have convinced us to focus on an architecture that enables close cooperation with other research groups in order to gain momentum for real applications. This paper will introduce a number of requirements for the next phase of development that enable the PowerMatcher to cope with new, future scenarios. The requirements lead to a number of architectural decisions that will support a more open software development trajectory.
\end{abstract}

Index Terms-- Multi-agent systems, Information technology, Distributed control, Power distribution, Power system control.

\section{NOMENCLATURE}

$\begin{array}{lll}\text { AMS } & - \text { Agent Management Service } \\ \text { CHP } & - & \text { Combined Heat and Power } \\ \text { DF } & - & \text { Directory Facilitator } \\ \text { DNO } & - & \text { Distribution Network Operator } \\ \text { ECN } & - & \text { Energy research Centre of the Netherlands } \\ \text { FIPA } & - & \text { Foundation for Intelligent Physical Agents } \\ \text { LMP } & - & \text { Locational Marginal Pricing } \\ \text { MAS } & - & \text { Multi-Agent System } \\ \text { VPP } & - & \text { Virtual Power Plant }\end{array}$

M.P.F. Hommelberg, C.J. Warmer, I.G. Kamphuis, J.K. Kok, (e-mail: \{hommelberg, warmer, kamphuis, j.kok\}@ecn.nl, work in the Intelligent Grids Program at the Energy research Centre of the Netherlands, Westerduinweg 3, P.O. Box 1, 1755 ZG Petten, The Netherlands.

B.J. van der Velde (email: Jorgen.van.der.Velde@ict.nl) Works at ICT Embedded, Kadijk 7 - 9747 AT Groningen

\section{THE POWERMATCHER - A SHORT INTRODUCTION}

\section{A. Market-based control using Multi-Agents}

I $\mathrm{n}$ market-based control, a large number of software agents are competitively negotiating and trading on an electronic market, with the purpose to optimally achieve their local control action goals. In [5] the first agent research applications and simulations carried out under the heading of market-based control were brought together. Most of the early research was aimed at climate control in office buildings with many office rooms, where local control agents compete in the allocation of cool (or hot) air.

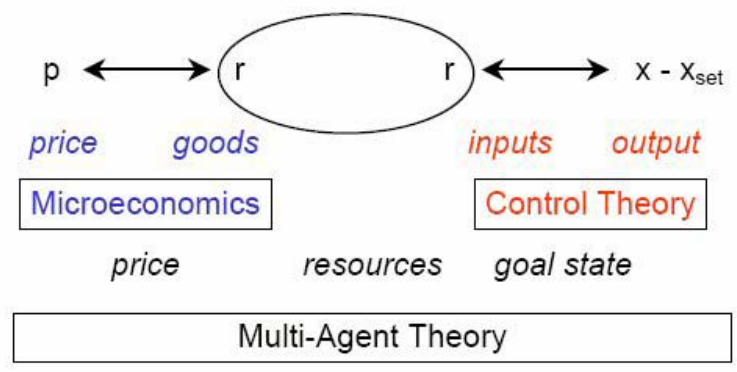

Figure 1 Microeconomics and control theory unified in a MAS

Recently, a systems-level theory of large-scale intelligent and distributed control was formulated [6], [7]. This theory unifies microeconomics and control theory into a multi-agent system (see Figure 1), and incorporates the agent research applications and simulations as described above. A central result is the derivation of a general market theorem that proves two important properties about agent-based microeconomic control: (1) computational economies with dynamic pricing mechanisms are able to handle scarce resources for control adaptively in ways that are optimal locally as well as globally ('societally'); (2) in the absence of resource constraints the total system acts as a collection of local independent controllers that behave in accordance with conventional control engineering theory.

\section{B. The PowerMatcher concept}

The PowerMatcher is a control concept for coordination of supply and demand in electricity networks with a high share of distributed generation, that implements the above marketbased control theory. It is concerned with optimally using the 
flexibility of electricity producing and consuming devices to alter their operation in order to adapt to the current energy value and the state of the underlying electricity network.

In the PowerMatcher concept each device is represented by a device agent, which tries to operate the process associated with the device in an economically optimal way. The electricity consumed or produced by the device is bought, respectively sold, by the device agent on an electronic exchange market [1], [2], [3]. The electronic market is implemented in a distributed manner via a network structure in which so-called PowerMatchers, as depicted in Figure 2, coordinate demand and supply of a cluster of devices directly below it. The PowerMatcher in the root of the tree performs the price-forming process; those at intermediate levels aggregate the demand functions of the devices below them. A PowerMatcher cannot tell whether the instances below it are device agents or intermediate PowerMatchers, since the communication interfaces of these are equal. This ensures a standardised interface for all types of devices.

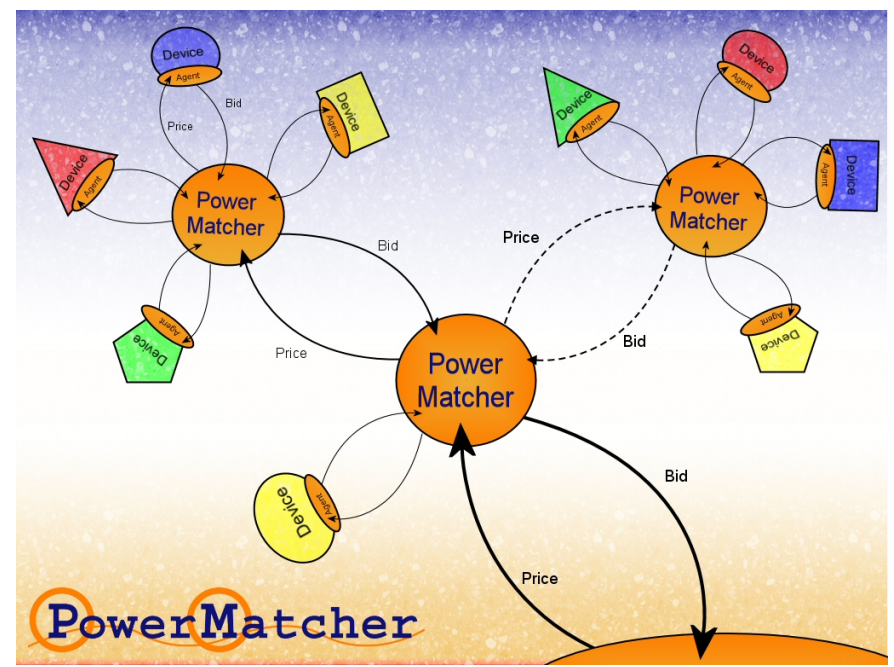

Figure 2 The PowerMatcher architecture; coming from a central tree based mechanism, growing towards a more organic, internet-like structure.

A PowerMatcher can be seen as a market agent that provides a coordination service for the connected suppliers and consumers of electricity. The root PowerMatcher has one or more associated market protocol definitions, which define the characteristics of the markets, such as the time slot length, the time horizon, and a definition of the execution event (e.g. "every 5 minutes", "every day at twelve o' clock"). When an execution event occurs, the root PowerMatcher sends a request to all directly connected agents (both intermediate market agents and device agents) to deliver their bids. The device bids are aggregated at the intermediate markets and passed on up-wards. The root PowerMatcher determines the market equilibrium price, which is communicated back to the intermediate market agents and from there to the device agents. From the market price and their own bid function each device agent can determine the power allocated to the device.

\section{SMART MARKETS}

\section{A. Event based markets}

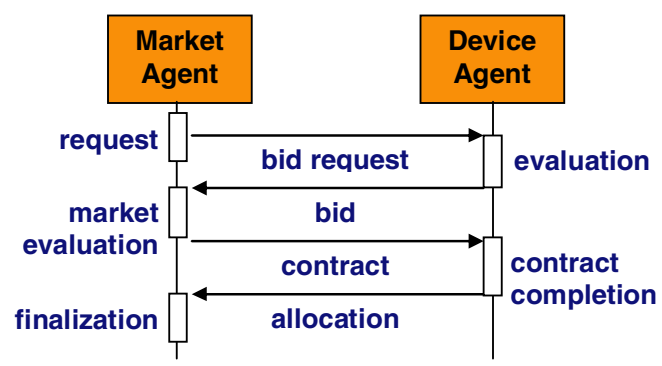

Figure 3 Agent interaction protocol for power exchange markets.

The PowerMatcher concept is mimicked after a power exchange market. This type of market has a structured market protocol (Figure 3) in which amounts of electricity are traded for market periods at discrete time intervals. Clearing of the market takes place some time before the trading period commences. The PowerMatcher market period typically is in the order of 1 to 15 minutes. Clearing of the PowerMatcher market is in the order of seconds. Although near-real-time control can be exerted in this way the fixed market periods cause some problems in real-time operation. It is paramount that process events - such as turning on the thermostat trigger a direct response from a device in order to fulfill user's demand. In the current scheme the market outcome will be disturbed until the next market period arrives. To overcome this effect the event based market concept has been developed.
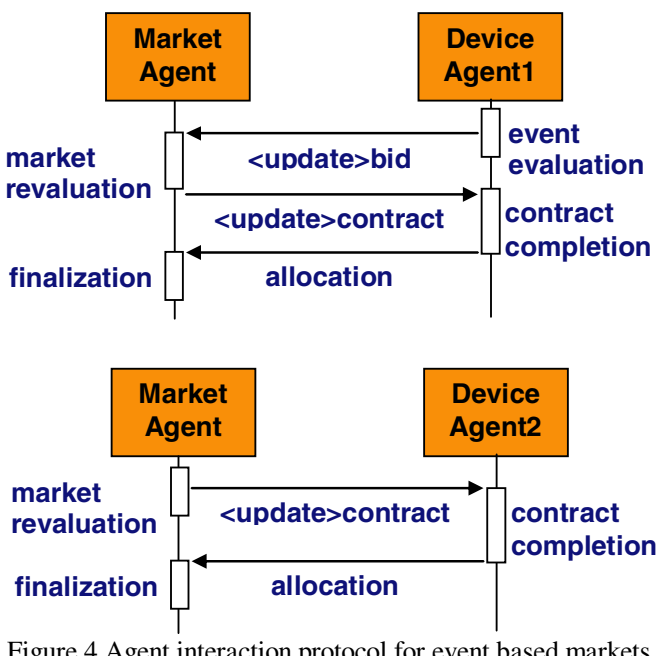

In an event based market agents can bid, or adjust their bid, at every moment their situation changes and directly receive a new contract after revaluation of the market. Also those agents that are influenced by the market revaluation are informed of their contract changes, as depicted in Figure 4. The event based design increases responsiveness of devices to events from their environment. Also the communication overhead may be reduced, especially for devices at rest (space heating during the night). And not all communication will be 
focused on fixed moments in time, but will spread out.

Event based markets may also give a means to handle ramping up and ramping down effects of devices. During ramping up a device may place new a bid on the market every few minutes, each time with a higher power bid, until maximum power is reached. Although this may lead to communication overhead (several bids are needed for one on/off action of a device), an enhanced bid protocol may be used that takes into account the ramping up/down cycle of a device.

Note that event based markets and periodic markets can be used in a hybrid way. The periodicity of the latter can be reduced if event based bids are allowed in the market. Also no special mechanism for detection of lost agents is needed since the periodic market will find out. The event based market concept also closely resembles the Napster or Kazaa networks, since only agents will be approached that are involved in a transaction. Thus market agents more and more take the role of broker agents.

\section{B. Market Discovery and Agent Subscription}

Each matcher in the PowerMatcher concept can be seen as an agent platform. In order to be FIPA-compliant an Agent Management System (AMS) is needed at each platform. The PowerMatcher offers such a system, which we have called the subscriber. The two platform services, subscribing and matching, are currently performed by the same entity or agent, which may have been a logical choice for periodic markets that are initiated by the matcher. The event based market, at which agents can initiate event based bids, may require a decoupling between the two mentioned services. The market agent just has to check that only agents, registered by the AMS, can place bids on its market.

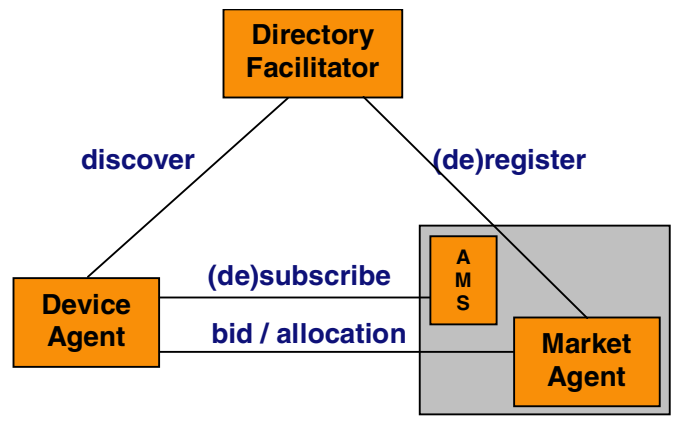

Figure 5 Market discovery and agent subscription protocol

For device agents to discover at which markets they can subscribe yellow page functionality has to be provided. For this functionality we plan to create a directory facilitator service at which all market agents can register their services. For network-coupled services part of the service description will be the location in the network in order for device agents to discover the right market to subscribe to. Device agents should subscribe to their own building network or substation network and not with neighboring networks. For commercial services device agents may be free to subscribe at a commercial market of their choice.

\section{Multi-commodity Matching}

The principle of demand and supply coordination can also be applied to other commodities than electricity, such as gas or heat and cold. This particularly holds for the building environment in which thermal buffers are available to a large extent [8]. Device agents that are operating on multiple commodity markets have to take into account the price of all commodities involved (e.g. a heat pump having to relate the value of electricity against the value of the generated heat and/or cold). Several issues have to be addressed:

- Real-time pricing: In PowerMatcher demonstrations the price range has always been artificial. A next step that has to be taken is to work with real prices in order to enable realtime pricing. This also makes it possible to weigh the different prices for different commodities. Processes that convert one commodity into another can relate the value of different commodities based on marginal cost.

- Price range propagation: The PowerMatcher concept uses fixed price ranges. The price range is communicated to the agents in a price protocol. Price range propagation is needed to 'translate' e.g. electricity price ranges into heat price ranges.

- Multiple markets versus one integrated market: In one integrated market an integrated valuation of all commodities can be made and an optimal market solution can be determined for trading of different commodities. However different commodities are mapped to different infrastructures and if network issues have to be taken into account in the market, such as capacity constraints, integrated markets lead to whole system optimisation, and one looses the flexibility of the distributed agent concept. So separate markets need to be addressed on which the different commodities are traded. The problem of interdependency between commodities can either be solved by introducing negotiations or by using prediction models for the separate markets.

Another way of dealing with separate markets is the introduction of decommitment of contracts. Decommitment is 'the action of foregoing a previous contract for another (superior) offer' [9]. This allows agents to increase their utility levels if one of the markets gives an unexpected result, e.g. if a heat pump promises to deliver heat while electricity prices suddenly rise.

\section{Constraint Handling}

A number of different architectures may be derived from the general PowerMatcher concept in Figure 2, in which different matchers can have different responsibilities such as preserving local network constraints, leading to different price-forming scenarios (cf. locational marginal pricing (LMP).

In order to prevent violation of network constraints several approaches may be taken. One method introduces so called guardian agents that become active in a local market in order to suppress peak load behaviour. This can be achieved by 
raising the commodity price to a high level. This may lead to scenarios for critical circumstances that evoke demand response behaviour from the device agents. Another approach has been described in [10], in which a theoretical framework and an algorithmic method are described for finding transport network feasible solutions in market-based flow resource allocation. A simpler algorithm can take care of single node constraints, such as maximum connection capacity in a building, by cutting off a bid function before passing it to a higher network level. It can be proven that this approach yields the same results as an LMP based market implementation.

\section{SMart Agent Design}

\section{A. Plug-and-play functionality}

Plug and play can be defined as a system feature that allows the addition of new elements in the system without requiring reconfiguration. This means that from its creation an agent shall be able to enter or leave a market without disturbing its operation. The DF and AMS (Figure 5) can provide the necessary services for the self-configuration of agents and the agent - market interaction.

Plug and play however in the context of local control should also safeguard local operation of a device, whether the agent is alive and/or is operating in a market or not. Some experience has been obtained with implementation of a robust back-up control for residential combined heat and power ( $\mu$ CHP) in one of the PowerMatcher field tests [11].

\section{B. Business agents}

Device agents have their own goals, based on their internal processes and optimization of the cost-benefit. Apart from the device agents also an other type of agent may participate in the market, having a business goal: the business agent.

At each level in the PowerMatcher network a business agent can input their goals at PowerMatcher nodes in the form of a standardized bid function. Thus a Distribution Network Operator (DNO) may trigger demand (and supply) response actions in a PowerMatcher market to avoid peak loads. This has been successfully demonstrated in a field test described in [11]. A virtual power plant operator may trigger load and supply based on the VPP operational state. Thus distributed devices can participate either in central power markets or become part of a balancing service. The latter has been subject of study in a field test described in [1]. Although the cluster can be controlled to reach a common goal, each single device agent remains autonomous, controlling its own environment.

\section{Hybrid real-life and simulation environment}

The PowerMatcher has been tested in several real-life field tests. Since the PowerMatcher is also used in a research environment, the design should support large simulations as well as a combination of both. Therefore an agent shall provide a simulation mode, offering functionality to start and control simulation of a device.

\section{Agent Learning Models Library}

Agents can apply intelligence based on a combination of environmental knowledge and historical patterns. Predictions can be used to create an agent planning strategy.

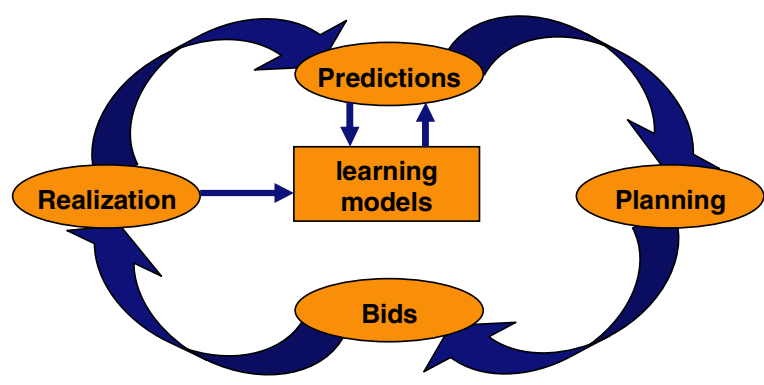

Figure 6 Building intelligence into agents.

The agents have been designed in such a way that a learning algorithm library can provide means for pro-active behaviour. Up to now the library contains persistency models and pattern recognition of recurrent fluctuations of environmental and market information.

\section{STANDARDIZATION ISSUES}

The device agents have been developed within the context of a market-based control application. Within this area agents are free to operate on any type of PowerMatcher organized market. Decoupling the agents from this area leads to a focus of agents that control their environment using sensors and actuators. The PowerMatcher service is just a service for which the control agent has implemented an interface. But the agents may be capable of much more: taking part in other control actions, e.g. demand response, or delivering services to other applications, e.g. information exchange on behalf of remote maintenance. The design of the device agents should provide the flexibility to add these options in a later stage. Also this may require a focus on interoperability with other power system applications. The FIPA standards [12] may be a suitable vehicle for such interoperability support. In this paper we already have complied to some FIPA terminology. Other FIPA issues that may be addressed are communication languages, compliance to content languages and ontologies.

\section{TOWARdS A POWERMATChER COMMUNITY}

The PowerMatcher concept can be characterized as a flexible and scalable concept that can be applied in a large variety of business cases: imbalance reduction, peak load reduction, virtual power plants, participation in a power exchange market or in ancillary services, micro-grid operation. This broad scope leads to a first conclusion that participation of other research groups and market parties is desirable in the next phase of development. The business potential for the different application fields has to be quantified. Development of a message exchange standard is needed in order to allow different market parties to develop 
their own intelligent agents.

At the time of writing of this paper we are still in discussion on how to create this critical mass for application of the PowerMatcher concept in real life. One option is to create a PowerMatcher Platform to address the above issues. Research parties may become involved in further conceptual development using a restricted open source model for the software. Market parties can reflect on standardization issues.

\section{CONCLUSION}

This paper introduces a robust framework for a multi-agent system aimed at market based coordination of supply and demand in future electricity grids with a large share of distributed generation. It does not give complete solutions for each issue addressed but serves as a blueprint in order to develop a 'next version' of the PowerMatcher concept which is robust with respect to future developments. It also serves as a guide book for future research, which is aimed at reaching an integrated bottom-up and market-based control of energy flows in the built environment. The need for interoperability with related power services has been identified, as well as the cooperation with the research community and market parties in order to develop a widely accepted standard for message exchange.

\section{REFERENCES}

[1] J.K. Kok, C.J. Warmer, I.G. Kamphuis, "PowerMatcher: Multi-agent control in the electricity infrastructure", AAMAS 2005 - 4th International joint conference on Autonomous Agents and MultiAgent Systems, Utrecht, July 2005 .

[2] J.K. Kok, C.J. Warmer, I.G. Kamphuis, "The PowerMatcher: Multi-agent Control of Electricity Demand and Supply", In M. Pechoucek and S.G. Thompson, "Agents in Industry: The Best from the AAMAS 2005 Industry Track, IEEE Intelligent Systems, March/April 2006.

[3] The PowerMatcher has its own website: http://www.powermatcher.net/

[4] S.D.J. McArthur et al, "Multi-agent systems for power engineering applications - Part I: Concepts, approaches and technical challenges; and Part II: Technologies, standards and tools for building multi-agent systems", IEEE Transactions on Power Systems, Vol. 22, p. 1753-1759, November 2007.

[5] S.H. Clearwater (Ed.), "Market-Based Control - A Paradigm for Distributed Resource Allocation". World Scientific, Singapore, 1996.

[6] J.M. Akkermans, J.F. Schreinemakers, J.K. Kok, "Microeconomic Distributed Control: Theory and Application of Multi-Agent Electronic Markets", Proceedings of CRIS $2004-2$ nd International Conference on Critical Infrastructures, Grenoble, 2004.

[7] J.M. Akkermans, J.F. Schreinemakers, J.K. Kok, "Emergence of Control in a Large-Scale Society of Economic Physical Agents", Proceedings of the AAMAS'04 Conference, New York, 2004.

[8] René Kamphuis, Jarno Dogger, Frans Nieuwenhout - Energy markets driving control of small-scale energy storage in combination with distributed co-generation. Proceedings of the IRES II Conference, Bonn, 2007.

[9] P.J. 't Hoen, V. Robu, J.A. La Poultré. Decommitment in a competitive multi-agent transportation setting. In: M Calisti, M. Klusch, R. Unland, (eds.), "Software Agent-Based Applications, Platforms and Development Kits". Birkhauser, Springer-Verlag, 2006.

[10] Koen Kok \& Hans Akkermans - Transport Network Feasible Solutions in Market-based Allocation of Flow Resources. in: Salvatore Pennacchio (ed.), Emerging Technologies in Robotics and Control Systems, , June 2007. ISBN: 978-88-901928-9-5.

[11] C.J. Warmer, M. P. F. Hommelberg, B. Roossien, J.K. Kok, J.W. Turkstra - A Field Test Using Agents for Coordination of Residential Micro-CHP. Proceedings of the ISAP Conference, Kaohsiung, 2007.
[12] Foundation for Intelligent Physical Agents (FIPA). See http://www.fipa.org/repository/standardspecs.html.

[13] P. Carlsson, "Algorithms for Electronic Power Markets", Ph.D. Thesis, Uppsala University, Sweden, 2004.

\section{BIOGRAPHIES}

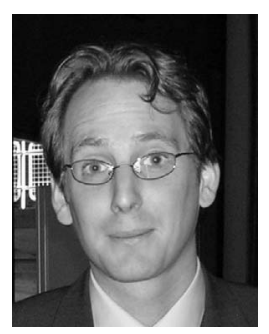

Maarten Hommelberg was born in Tilburg on October 9 1978. He graduated in the field of building services on the University of Technology in Eindhoven in 2005. The graduation subject was titled "Software agents for a building management system". His first and current employer is ECN. He is project leader in the Virtual Power Plant project and is currently working on optimizing power demand and supply flows in the distribution network using market based agent algorithms.

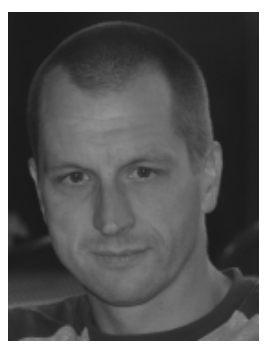

Jörgen van der Velde has 13 years of experience in hardware and software development. Since 2001 he works for ICT Embedded BV, mainly in the role of software architect. In this role he is responsible for the quality of the software product to be delivered, coaching of software developers, support of business development and communication within the team, with the customer and external parties. Main education: Engineering Physics (master degree) at the University of Groningen. Currently Jörgen works in the Energy and Utilities branch of ICT. $\mathrm{He}$ is member of the Smart Power System (http://www.smartpowersystem.com) Administrative Processes and ICT and Working Groups and NEN NTA8130 committee.

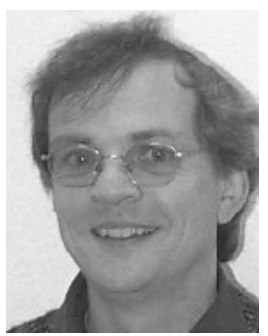

Cor Warmer was born in Utrecht on March 8 1955. He graduated as a mathematical statistician from the Universiteit van Amsterdam in 1981. He joined ECN in 1981 as a mathematics and statistics consultant in a scientific mainframe environment He was later involved in a large number of projects focusing on data and object modeling. His current research includes process optimization of large energy consuming systems and optimization of power demand and supply flows in the distribution network using market based agent algorithms.

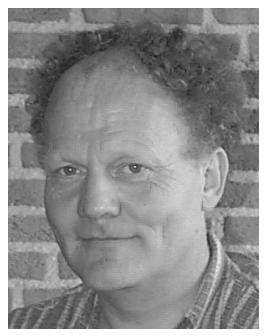

René Kamphuis was born in 1952. He graduated from Nijmegen University in 1976 in Chemistry. After that he got a Ph. D. from Groningen University in Chemical Physics. His employment experience at ECN started with a position at the computing center. From there he went to a number of software engineering positions. Since then he is involved in the Intelligent Energy Grids program at ECN. He has been involved in a number of projects concerning the application of agent technology for comfort management in buildings and dynamic distributed applications in the electricity network.

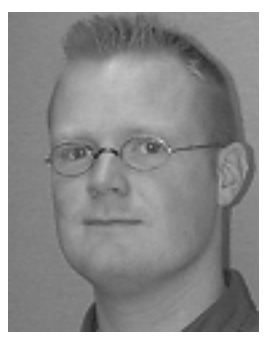

Koen Kok (1969, male) received Bachelor degrees in Electrical Engineering (1992) and Computer Engineering (1992). After a short working period at the University of Groningen, he started to study Computer Science at the same university and received his MSc in Computer Science in 1998. From 1998 to date, he is working as Researcher and Project Co-ordinator at ECN. He is working in the interdisciplinary field between electrical engineering, systems control and ICT. His current research focus is on intelligent distributed control mechanisms for electricity grids with ahigh penetration of distributed generation. 